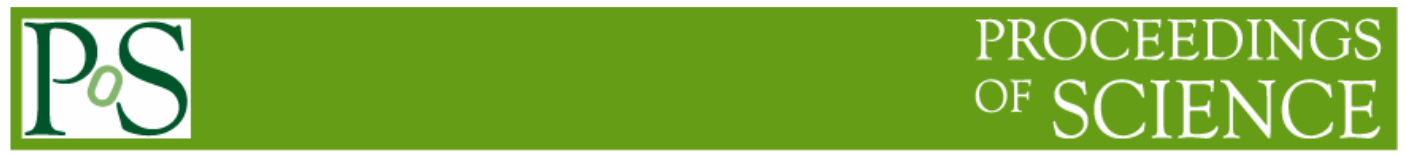

\title{
Super-grains: seeds of planet formation
}

\author{
Jane Greaves ${ }^{1}$ \\ University of St Andrews \\ Physics \& Astronomy, North Haugh, St Andrews, Fife, KY16 9SS, UK \\ E-mail: jsg5 - at - st-andrews.ac.uk
}

\begin{abstract}
Sensitive radio observations towards young stars can detect not only jets but also dust emission from a circumstellar disc. A reservoir of large dust grains is a sign that larger bodies are accumulating, on the road to planets. A 1-cm survey with the Green Bank Telescope shows that a number of discs in the Taurus and Ophiuchus star-forming regions have such signs of dust growth and so may be called 'proto-planetary'. Inclusion of the largest grains - which dominate the mass - boosts more discs into the regime of the Minimum Mass Solar Nebula, so future systems of gas giant planets are expected. The next step is to observe the discs with high resolution to see if the grain growth occurs in the regions at a few AU where planetary cores should form - eMERLIN will be very effective for this, and a Legacy survey is planned. Sample calculations show that even the zone of terrestrial planet formation could be detected and resolved.
\end{abstract}

From planets to dark energy: the modern radio universe University of Manchester, Manchester, UK

1-5 October, 2007

\footnotetext{
$1 \quad$ Speaker
} 


\section{Introduction}

The formation of rocky (terrestrial) planets and the cores of gas giants require the accumulation of refractory particles inherited from the interstellar medium. These dust grains are part of the protostellar envelope and then settle to the mid-plane of the circumstellar disc, from which material accretes onto the young star. A small residual is needed for example to form the planets of the Solar System - the rocky cores comprise $50 \mathrm{M}_{\text {Earth }}$ and so the Minimum Mass Solar Nebula (MMSN) must have contained at least $20 \mathrm{M}_{\text {Jupiter }}$ of gas and dust, assuming a primordial gas-to-dust mass ratio of 100 . One of the problems of observational planet formation studies is that many discs appear to be of comparatively low mass; for example over the Taurus and Ophiuchus star-forming regions, the typical disk only contains 5 Jupiter masses [1]. This is a problem for explaining the incidence of giant exo-planets, which long-term Doppler surveys suggest occur around $10-20 \%$ of stars, made worse by the fact that exo-planet systems detected so far are typically more massive than the sum of the Sun's planets.

One solution is that the exo-disc masses may have been underestimated. In particular, the refractory mass should be dominated by the largest grains, which are best detected in the radio. Most surveys of disc masses have been conducted at millimetre wavelengths, where the emission is reasonably bright (tens of mJy) and longwards enough that the blackbody or greybody dust emission is optically thin. However, millimetre-sized particles are poor emitters at wavelengths longer than their sizes - which means that if centimetre wavelength dust emission is detected, then grains of at least centimetre sizes are responsible. Such studies are difficult because greybody grain emission typically drops off at long wavelengths with a steep spectrum of roughly $v^{3}$, although this also makes it easy to distinguish from the rather flat spectrum of an ionised wind or jet in unresolved but multi-wavelength data.

The prototype disc showing large dust grains is TW Hya [2], which has a dust excess longwards of $7 \mathrm{~mm}$ compared to extrapolations from data at $\sim 1 \mathrm{~mm}$ and shorter. A $7 \mathrm{~mm}$ survey conducted with the VLA for Taurus stars [3] showed several examples of dust discs, and confirmed that the emission was spread out to large enough radii that the discs are indeed optically thin. The slope of the spectral energy distribution (SED) was in most cases shallower than $v^{3}$ which indicates that the largest grains are of a size amax greater than the observing wavelength - i.e. the spectrum is approaching the $v^{2}$ of a blackbody spectrum of large perfectly emitting particles. Detecting these large particles is a signpost that planet formation by accumulation into pebbles, boulders, planetesimals... may be underway. The growth to e.g. $10 \mathrm{~cm}$ bodies is already substantial compared to the sub-micron sizes of the inherited interstellar grains - a similar leap of a few $10^{5}$ would reach the sizes of asteroids.

\section{Results}

A more comprehensive study than [3] is needed to assess the typical masses of protoplanetary discs - most radio studies so far have picked known millimetre-bright discs in the expectation that these will also be the most bright in the centimetre. The results discussed here (preliminary analysis; Greaves et al. in prep.) are from a survey performed at the Green Bank Telescope in April 2007, which adopted a less biased approach. All the discs in Taurus and Ophiuchus that exceed $100 \mathrm{mJy}$ at $1.3 \mathrm{~mm}$ were observed, corresponding to current mass estimates of about an MMSN upwards. Thus, these discs should have some planet-forming capability, but many are 
below the few-MMSN boundary required to make core-growth calculations work, i.e. produce a Jupiter-like planet.

In all 30 stars were observed, of which around half show dust emission at 8-11 mm wavelengths (Ka band). For this half of the survey, the preliminary estimate is that the dust masses including large grains are raised by typically a factor of two. While this is not large, it makes a notable difference to planet-forming capability - the (millimetre-based) $\mathrm{M}_{\text {disc }}$ distribution is a wide lognormal function, so that probing a factor of two deeper down the highend tail would boost a significant number of discs into the few-MMSN regime.

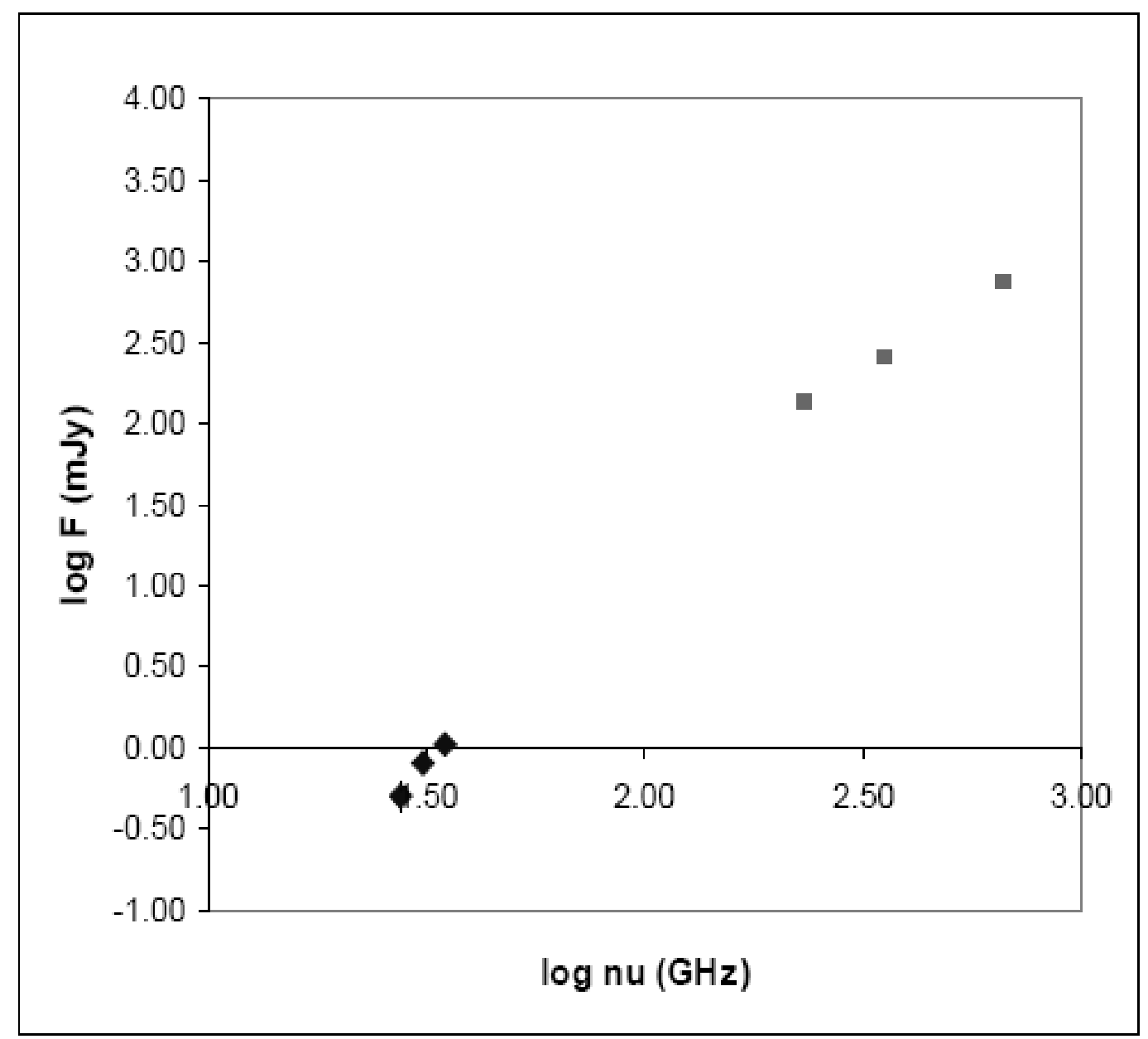

Figure 1. Example SED from the GBT disc survey.

A typical submillimetre-to-centimetre SED from the GBT survey is shown in Figure 1. Unlike the TW Hya SED, there is not a cm-excess of dust emission, extrapolating from the $\mathrm{mm}-$ datapoints on the right of Figure 1. However, the emissivity of large grains is poor, so that even this limited cm-flux boosts the total dust mass, and so the inferred mass of the disc assuming that it retains its primordial gas. The turndown in $\mathrm{cm}$-flux also suggests that the grains in this disc do not yet approach large perfect-emitter sizes - so this may be a useful diagnostic of how 
far grain growth has proceeded in different systems. Figure 2 shows a sketch of how the relative populations of moderate-sized and large grains could affect the submillimetre to centimeter SED. If grain growth has only turned a few mm-sized particles into $\mathrm{cm}$-sized bodies, then the disc may be bright in the millimetre, well-matched to the dominant population, but faint in the centimetre. If grain growth has used up most of the mm-sized particles already, the emitting surface is reduced for the cm-sized population, but a more continuous SED is expected.
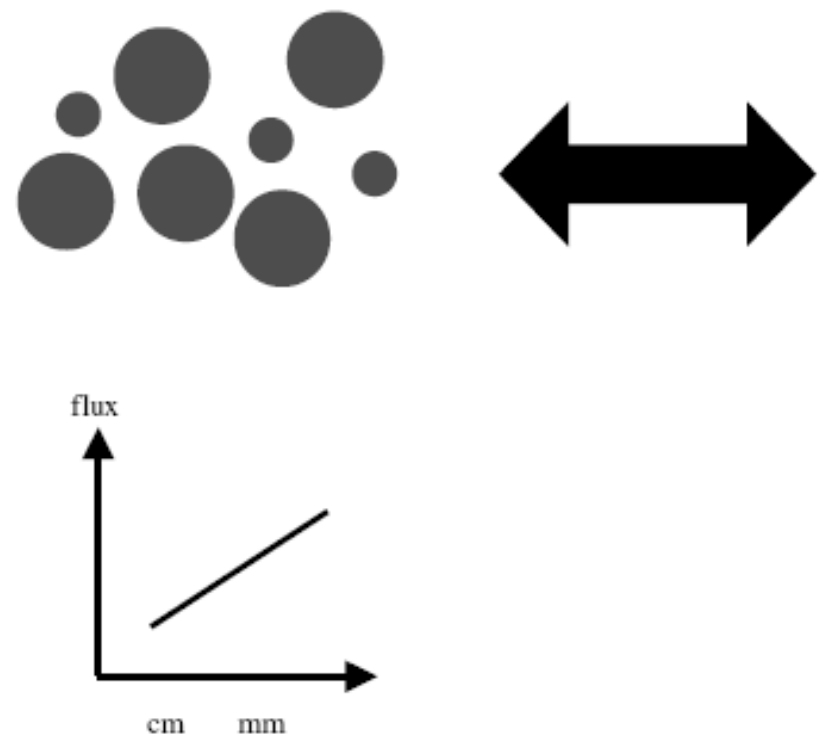

Figure 2. Sketch of spectral energy distributions for different amounts of grain growth. Relative numbers of large and small grains are illustrative only.

\section{Case study and Legacy prospects}

The GBT survey essentially used the telescope as a $100 \mathrm{~m}$.light bucket. and was very sensitive (below 1 mJy levels), making a survey of 30 discs possible in under 20 hours of observation. The next stage, having identified some promising .proto-planetary. discs, is to see where the grain growth is occurring, which requires resolving the discs. The Taurus and Ophiuchus regions are $\sim 150 \mathrm{pc}$ away, so the 5 -AU orbit of Jupiter subtends about 70 mas and the 1-AU orbit of the Earth just under 15 mas. If grains could be mapped over these small scales, it would be possible to see the formation of planetary cores in action. The gas giant formation phase is brief (probably $<10 \mathrm{Myr}$ ) and Taurus is the nearest region in the northern sky. However, the epoch of Earth formation is a few tens of Myr, and some of the more numerous moving groups and local associations of stars fall in this timespan. Then discs imaged at closer distances of say 30 pc would need only $\sim 70$ mas resolution to examine the scale of the Earth's orbit.

Thus an eMERLIN survey of planet formation is very attractive. At C-band, 40 mas resolution would place approximately two beams across the orbit of Jupiter in Taurus or the orbit of the Earth around a moving-group star $30 \mathrm{pc}$ away. Such observations at around $5 \mathrm{~cm}$ would trace grains up to tens of centimetres in size. A sensitivity of $2 \mu \mathrm{Jy}$ in a 12-hour track corresponds to 
approximately $1 \mathrm{M}_{\text {Earth }}$ of dust at $1 \mathrm{AU}$ as seen from $30 \mathrm{pc}$, assuming an Earth-like temperature and a dust opacity $\sim 0.01 \mathrm{~cm}^{2} / \mathrm{g}$ (see Figure 4 of [4] for $\mathrm{a}_{\max } \sim 10 \mathrm{~cm}$ ). This corresponds to the lower bound of refractory mass needed to make our planet.

A Legacy survey with eMERLIN could therefore for the first time resolve scales as small as those of terrestrial planet formation and also detect the tiny amounts of disc material needed. By the time of eMERLIN observations, a large number of candidate discs will have been found from radio surveys with GBT and VLA or via the far-infrared disc indicators from facilities such as Spitzer and SCUBA-2. A Legacy survey could therefore target a moderate number of the most promising proto-planetary discs, and image the formation of planets like the Earth and Jupiter in action.

\section{References}

[1] S. M. Andrews, J. P. Williams, A submillimeter view of circumstellar dust disks in $\mathrm{r}$ Ophiuchus, (2007) [arXiv: 0708.4185]

[2] N. Calvet et al., Evidence for a developing gap in a 10 Myr old protoplanetary disk (2002), Astrophys. J. 568, 1008

[3] J. Rodmann et al., Large dust particles in disks around Taurus stars (2006), Astron. Astrophys. 446, 211

[4] Draine B. T., On the submillimeter opacity of protoplanetary disks (2006), Astrophys. J. 636,1114 